
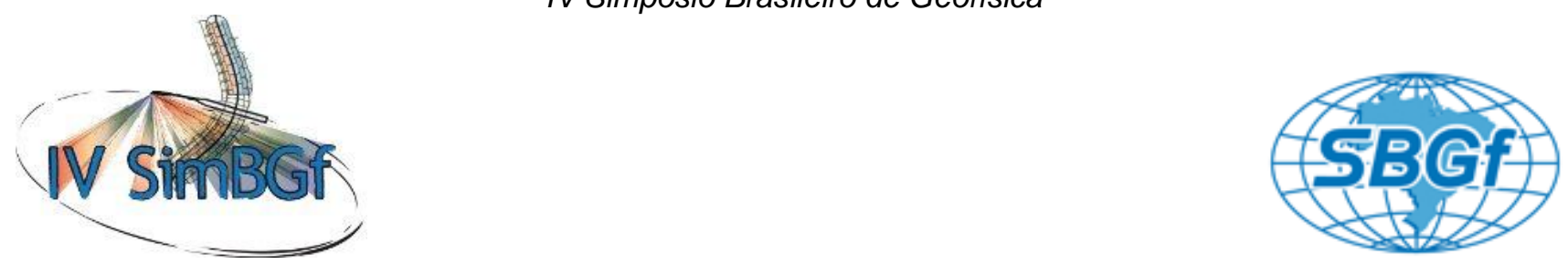

\title{
Tensor Gradiente Gravimétrico da Bacia do Paraná
}

\section{Henrique Bueno dos Santos ${ }^{\star 1}$, Naomi Ussami ${ }^{1}$ \& Nelsi Côgo de Sá1, IAG - USP.}

Copyright 2008, SBGf - Sociedade Brasileira de Geofísica

Este texto foi preparado para a apresentação no IV Simpósio Brasileiro de Geofísica, Belém, 14 a 17 de novembro de 2010. Seu conteúdo foi revisado pelo Comitê Técnico do IV SimBGf, mas não necessariamente representa a opinião da SBGf ou de seus associados. É proibida a reprodução total ou parcial deste material para propósitos comerciais sem prévia autorização da SBGf.

\section{Abstract}

The gravity gradient tensor (GGT) components of free-air and Bouguer gravity anomalies for the Paraná Basin were computed using land-based data and the Fourier transform GGT calculation technique. In order to avoid short wavelength gravity anomalies interference when calculating the derivatives, the original data was upward continued for different heights. The objective of this study is evaluate the amplitude of GGT components at different heights which is capable to detect mass heterogeneities within the crust and in the upper mantle. Free-air and Bouguer anomaly digital models of the Paraná Basin with spatial resolution of $5^{\prime} \times 5^{\prime}$ were computed using least squares collocation. This methodology involved a combination of global geopotential models, conventional land and marine gravity and topographic data. The GGT were calculated from free-air and Bouguer anomalies using the Fast Fourier Transform (FFT) technique of Laplace's equation proposed by Mikus \& Hinojosa (2001). The correct functioning of the computer program was checked by comparing the GGT predicted by analytical expressions of a right rectangular prism with the GGT obtained from the FFT-algorithm. At $10 \mathrm{~km}$ height the amplitude of GGT components range approximately from -5.0 to 5.0 Eötvös and decay to -1.0 to 1.0 Eötvös at 100 $\mathrm{km}$ altitude. The vertical component of GGT highlight important lithospheric features such as the continental margins and an intracontinental suture zone which separates major cratonic units such as the São Francisco and Amazon cratons.

\section{Introdução}

De posse de modelos gravimétricos com alta resolução é possível realizar estudos crustais e litosféricos de grandes áreas como, por exemplo, a Bacia do Paraná, região que apresenta uma boa cobertura de dados de gravimetria terrestre.

Outra utilidade desses mapas é de poder simular a gradiometria gravimétrica com a variação da altitude. As anomalias ar-livre e Bouguer da Bacia do Paraná foram calculadas para diferentes altitudes e para cada altitude de continuação foram determinados as componentes do tensor gradiente gravimétrico (TGG) foram estimadas utilizando a solução proposta por Mickus \& Hinojosa (2001).

A partir de dados disponíveis na região $10 S$ - 40S e $65 \mathrm{~W}$ - 35W,que resultaram de levantamentos terrestres convencionais e missões espaciais, foram determinados modelos digitais de alta resolução ( $5^{\prime}$ x 5') das anomalias gravimétricas. A metodologia envolveu a combinação de modelos geopotenciais, dados gravimétricos e topográficos para a representação do campo de gravidade. O processamento para cada tipo de dado envolveu três etapas: 1) homogeneização, avaliação e reamostragem dos dados básicos; 2) junção dos dados; e 3) determinação de modelos na forma digital. Com o modelo geopotencial de alto grau EGM96, o campo de gravidade foi representado por meio da componente determinística, calculada com os coeficientes do EGM96 ( $n=360$ ); e da componente estocástica, determinada com o uso da Colocação por Mínimos Quadrados, usando a aproximação local. As covariâncias da componente estocástica foram calculadas a partir de anomalias gravimétricas, através da densidade espectral de potência, e representada pelo modelo local desenvolvido por Forsberg (Sá, 2004).

A Figura 1 mostra o mapa de anomalia Bouguer com resolução de $5^{\prime}$ x 5'. Os números indicam as principais províncias tectônicas da região.

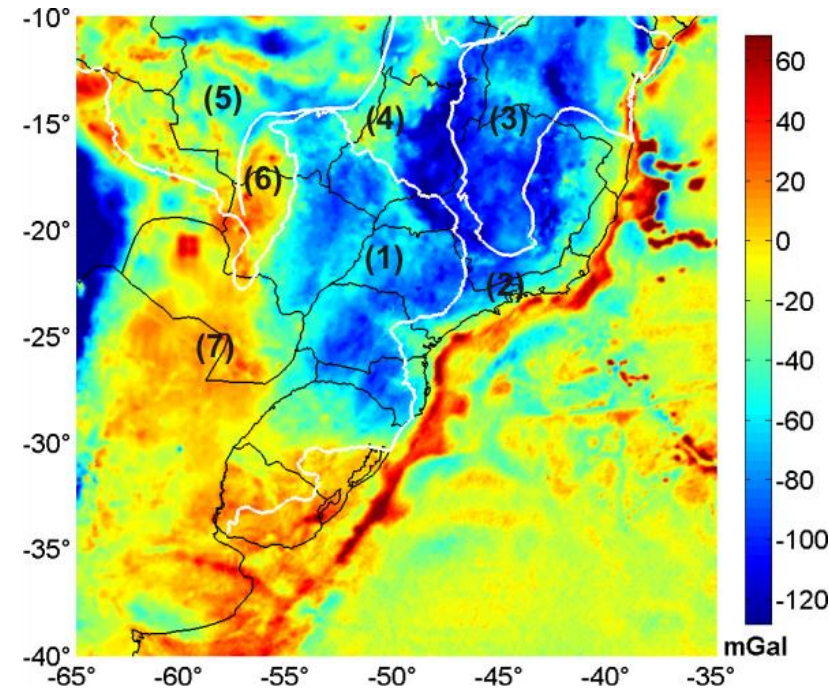

Figura 1 - Anomalia Bouguer da Bacia do Paraná. Os números indicam as principais províncias tectônicas da região: (1) Bacia do Paraná; (2) Província Mantiqueira; (3) Cráton São Francisco; (4) Provincia Tocantins; (5) Cráton Amazonico; (6) Bacia do Pantanal; (7) Bacia do Chaco-Paraná; 
De posse desse modelo digital calcularam-se a continuação para cima para 10, 30, 50 e 100 km (Figura 2).

\section{Continuação para cima da anomalia Bouguer $\left(g_{z}\right)$}

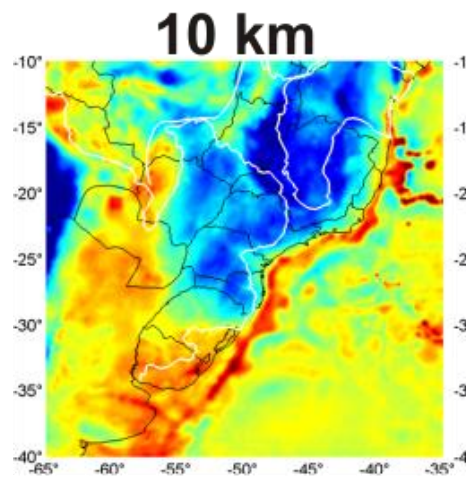

(a)

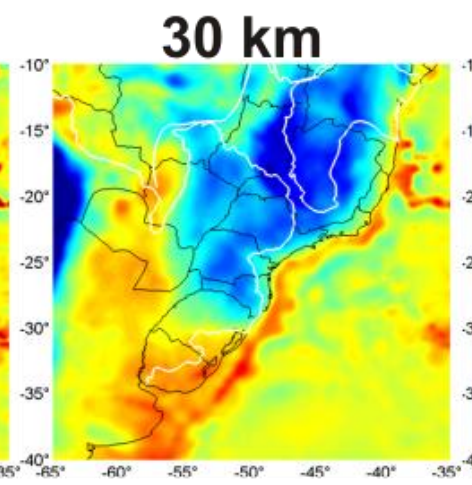

(b)

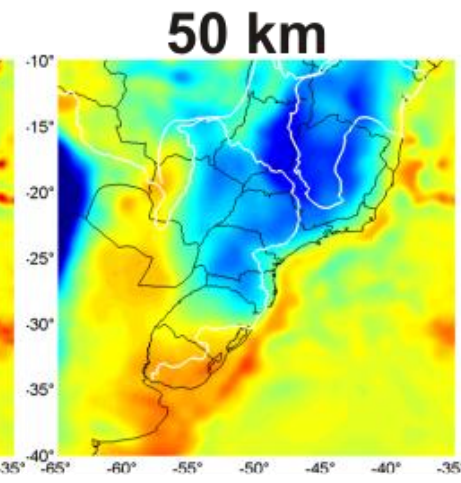

(c)

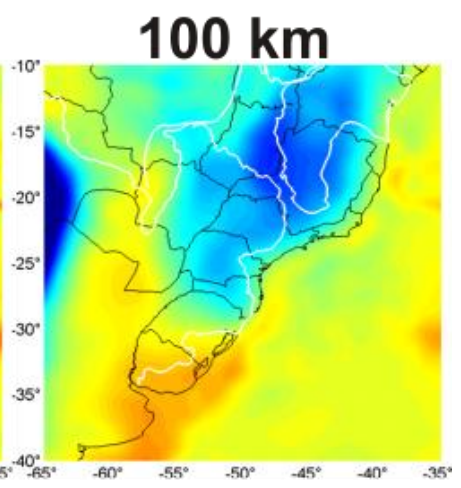

(d)

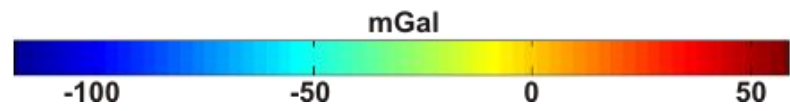

Figura 2 - Continuação para cima (mGal) da anomalia Bouguer para diferentes altitudes: 10, 30, 50 e $100 \mathrm{~km}$.

\section{Cálculo do Tensor Gradiente Gravimétrico (TGG)}

Os dados disponíveis para esse processamento são as anomalias ar-livre e Bouguer. As derivadas da componente vertical $\left(g_{z x}, g_{z y}, g_{z z}\right)$ da aceleração da gravidade são as componentes do TGG facilmente obtidas através da solução da equação de Laplace em coordenadas cartesianas.

Mickus \& Hinojosa (2001) mostraram como calcular as componentes do TGG (Figura 3) partindo dos dados préexistentes da componente vertical da aceleração de gravidade.

O tensor é uma matriz $3 \times 3$ (equação 1), onde cada elemento é a taxa de variação de um das componentes do vetor aceleração nas outras três direções. De acordo com a equação de Laplace, para um campo externo à distribuição de massas, a soma das componentes diagonais é zero, $g_{x x}+g_{y y}+g_{z z}=0$. Além disso, a matriz é simétrica em relação à diagonal, ou seja, $g_{x y}=g_{y x}, g_{x z}=$ $g_{z x}$ e $g_{y z}=g_{z y}$. Esses dois fatos implicam que somente cinco componentes do TGG são independentes. Por exemplo, conhecendo as componentes $g_{x x}, g_{y y}, g_{z z}, g_{x z} e$ $g_{y z}$, podem ser determinados as quatro componentes restantes, da seguinte forma:

$$
T G G=\left[\begin{array}{lll}
g_{x x} & g_{x y} & g_{x z} \\
g_{y x} & g_{y y} & g_{y z} \\
g_{z x} & g_{z y} & g_{z z}
\end{array}\right]
$$

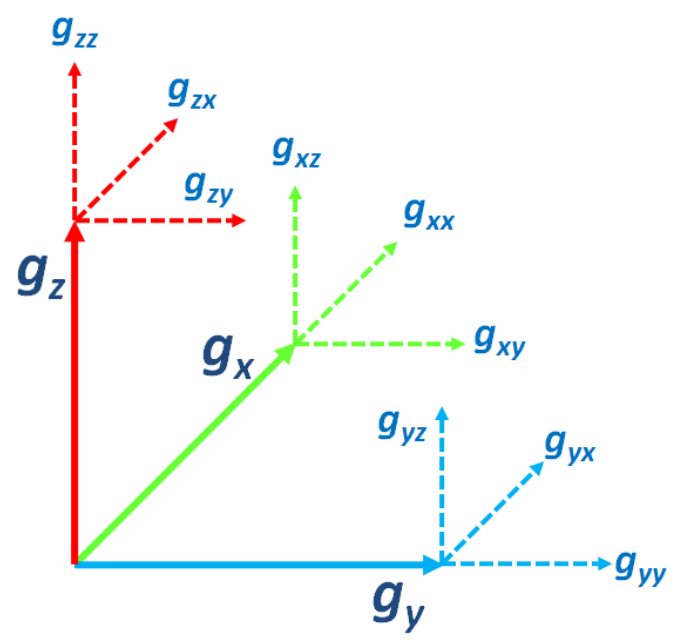

Figura 3 - Orientação do TGG. As cores, azul e verde, ilustram as componentes horizontais do TGG, enquanto o eixo vermelho mostra a componente vertical do TGG. $x, y$ e $z$ estão orientados respectivamente para o norte, leste e para cima.

As componentes do TGG também são de grande importância para os estudos crustais e litosféricos. Com elas é possível determinar, por exemplo, o centro de massa dos corpos $\left(g_{x z}\right.$ e $\left.g_{y z}\right)$, seus limites laterais $\left(g_{x x} e\right.$ $\left.g_{y y}\right)$ e direção preferencial de estruturas de densidade $\left(g_{x y}\right)$. A componente vertical $\left(g_{z z}\right)$ fornece diretamente a posição espacial e é correlacionada mais facilmente com a geologia de subsuperfície (Braga, 2006) realçando estruturas muitas vezes não observadas ao analisar apenas a componente da aceleração de gravidade $g_{z}$.

O algoritmo de Mikus \& Hinojosa (2001) foi implementado computacionalmente para o cálculo das componentes do 
TGG. Para conferir a correta implementação do programa, utilizaram-se dados de TGG sintéticos gerados por um corpo prismático. Em seguida, calculou-se o TGG para as anomalias ar-livre e Bouguer da bacia do Paraná para a altitude de $10 \mathrm{~km}$ (Figura 4). Esta altitude foi escolhida arbitrariamente a fim de evitar efeitos de ruídos e amplificação de anomalias de curto comprimento de onda que podem mascarar os sinais da derivada associados às fontes crustais e litosféricas. Isso possibilitará estudar a interferência dos curtos comprimentos de onda e o comportamento as anomalias com a altitude.

\section{Discussão e Conclusões}

A Figura 5 mostra o comportamento da derivada vertical da anomalia Bouguer e da sua derivada vertical para 10, 30,50 e $100 \mathrm{~km}$ de altitude. Nota-se que mesmo com a continuação para cima para $100 \mathrm{~km}$ (Figura 5d), o que implica na atenuação das anomalias com curtos comprimentos de onda, ainda é possível mapear anomalias importantes de escala litosféricas, como zonas de sutura e anomalias gravimétricas na margem continental (Figura 5d). Além disso, há predominância de anomalias negativas em quase todo o continente,

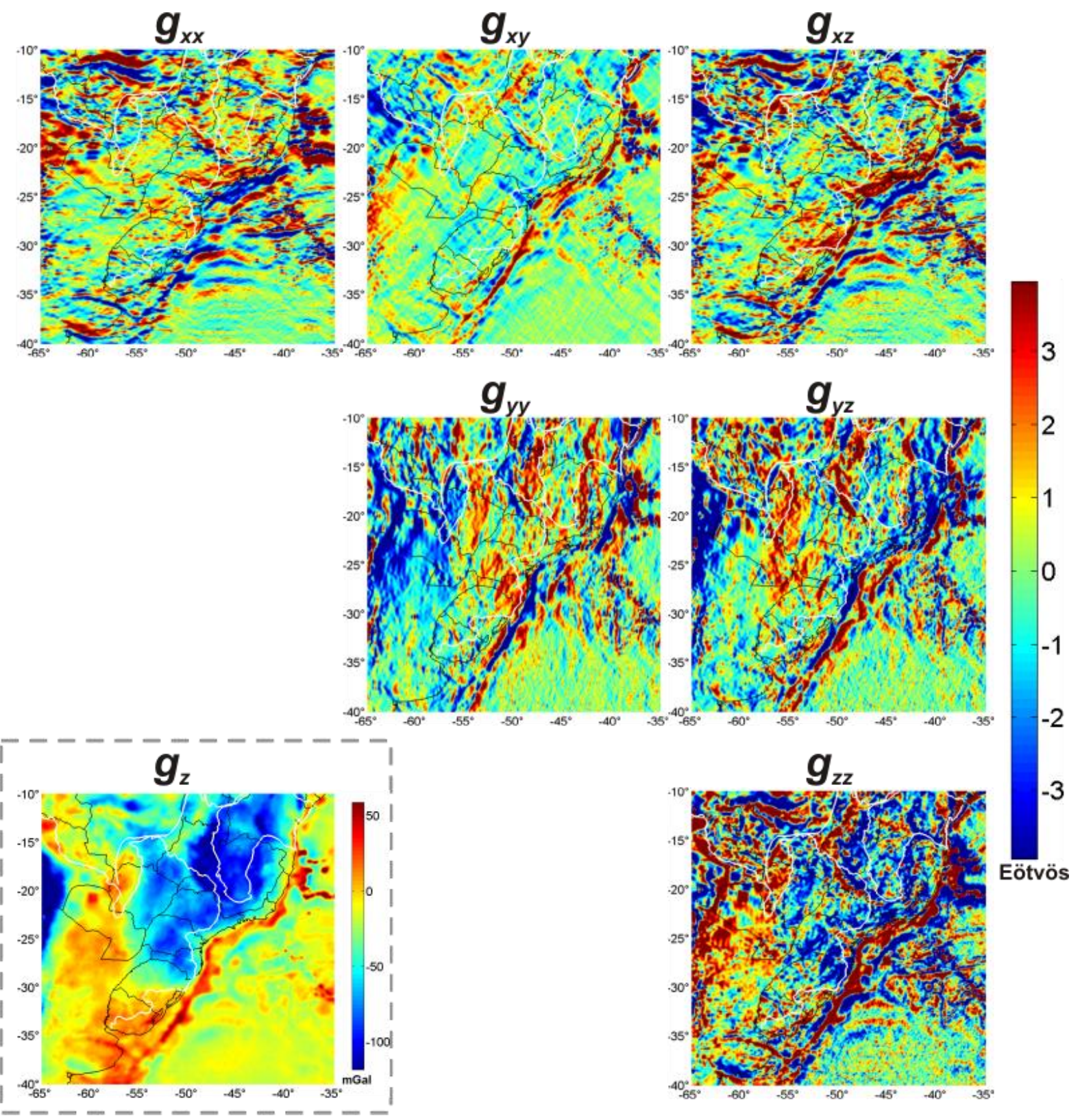

Figura 4 - TGG da anomalia Bouguer continuado para $10 \mathrm{~km}$. A figura mostra seis componentes do TGG: $\boldsymbol{g}_{\boldsymbol{x} x}$, $\boldsymbol{g}_{\boldsymbol{x y}}$, $\boldsymbol{g}_{x z}, g_{y y}, g_{y z}$ e $g_{z z}$ onde cinco deles são independentes, pois uma das componentes da diagonal principal pode ser obtida a partir das outras. 


\section{Derivada vertical $\left(g_{z z}\right)$}

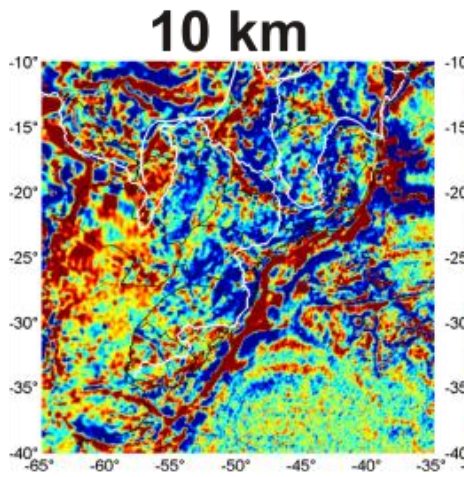

(a)

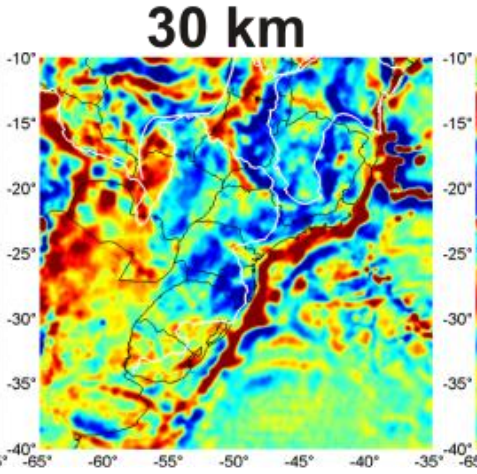

(b)
$50 \mathrm{~km}$

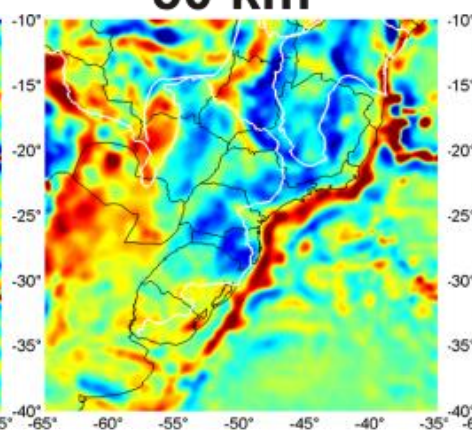

(c)
$100 \mathrm{~km}$

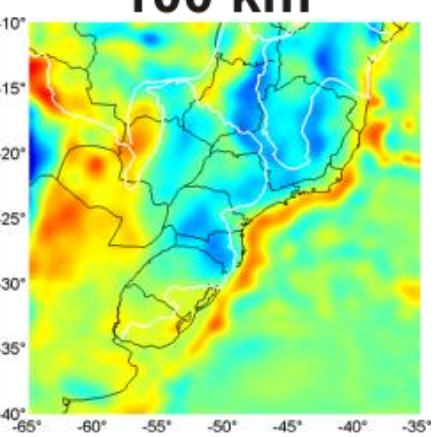

(d)

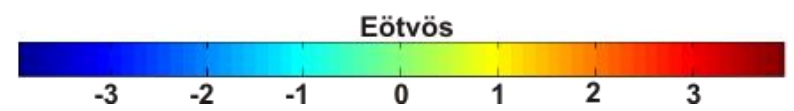

Figura 5 - Derivada vertical em Eötvös $\left(10^{-4} \mathrm{mGal} / \mathrm{m}\right.$ aproximadamente) da anomalia Bouguer para diferentes altitudes: $10,30,50$ e $100 \mathrm{~km}$.

principalmente na região da bacia do Paraná e Cráton São Francisco. Litosferas cratônicas são caracterizadas por gradientes verticais negativos. Além disso, mesmo a $100 \mathrm{~km}$ de altitude a derivada vertical da anomalia Bouguer indica forte correlação entre gradientes positivos e zonas de sutura, como na borda do Cráton São Francisco, e underplating basáltico na crosta inferior, na Bacia do Paraná.

Finalmente, os resultados desse trabalho mostram que o TGG, amplamente utilizado para identificar variações de densidade de fontes rasas, provou ser igualmente útil no mapeamento de densidade na crosta terrestre em grande escala e distribuição de densidade na litosfera.

\section{Agradecimentos}

Ao CNPq (Centro Nacional De Desenvolvimento Científico e Tecnológico) pela bolsa de mestrado para $\mathrm{H}$. B. Santos. Este projeto está sendo apoiado por recursos do Projeto Temático da FAPESP coordenado por Ícaro Vitorello (processo 2009/50493-8).

\section{Referências}

Braga, A. B., 2006. Modelagem numérica e validação de dados tensoriais de aerogradiometria gravimétrica $3 D$ FTG. Dissertação de Mestrado, Instituto de Geociências, da Universidade Federal do Rio de Janeiro, Rio de Janeiro, 17-18.

Mickus, K. L., Hinojosa, J. H., 2001. The complete gravity gradient tensor derived from the vertical component of gravity: a Fourier transform technique. Journal of Applied Geophysic, vol. 46: 159-174.

Sá, N. C. de, 2004. O campo de gravidade, o geóide e a estrutura crustal na América do Sul - novas estratégias de representação. Tese de Livre Docência, Instituto de Astronomia, Geofísica e Ciências Atmosféricas da Universidade de São Paulo, São Paulo. 